\title{
dspace.vutbr.cz
}

\section{Pseudo-differential filter operating in current mode}

\author{
SLÁDOK, O.; VELIC, L.; KOTON, J.; HERENCSÁR, N.
}

Proceedings of 10th International Congress on Ultra Modern Telecommunications and Control Systems - ICUMT 2018

pp. 1-4

elSBN: 978-1-5386-9361-2

DOl: http://dx.doi.org/10.1109/ICUMT.2018.8631215

Accepted manuscript

(C2018 IEEE. Personal use of this material is permitted. Permission from IEEE must be obtained for all other uses, in any current or future media, including reprinting/republishing this material for advertising or promotional purposes, creating new collective works, for resale or redistribution to servers or lists, or reuse of any copyrighted component of this work in other works. Ondrej Sladok, Ladislav Velic, Jaroslav Koton, Norbert Herencsar " Pseudo-Differential Filter Operating in Current Mode", Proceedings of 10th International Congress on Ultra Modern Telecommunications and Control Systems - ICUMT 2018, pp. 1-4, 2018. DOI: 10.1109/ICUMT.2018.8631215. Final version is available at https://ieeexplore.ieee.org/document/8631215 


\section{Pseudo-Differential Filter Operating in Current Mode}

\author{
Ondrej Sladok \\ Department of Telecommunications \\ Brno University of Technology \\ Brno, Czech Republic \\ sladok@phd.feec.vutbr.cz \\ Jaroslav Koton \\ Department of Telecommunications \\ Brno University of Technology \\ Brno, Czech Republic \\ koton@feec.vutbr.cz
}

\author{
Ladislav Velic \\ Department of Telecommunications \\ Brno University of Technology \\ Brno, Czech Republic \\ xvelic03@stud.feec.vutbr.cz \\ Norbert Herencsar \\ Department of Telecommunications \\ Brno University of Technology \\ Brno, Czech Republic \\ herencsn@feec.vutbr.cz
}

\begin{abstract}
In the paper, the theory of pseudo-differential filters is examined and supported by designing a fourth-order frequency filter operating in current mode and implemented as cascade connection of two basic second-order blocks. The basic block employs three current conveyors as active elements and four passive elements. The filter is characterized by a high output impedance, and minimum number of passive elements, whereas capacitors are grounded. To prove the operability of the final filter, the partial second-order pseudo-differential filters were interconnected in four different ways. The simulation results of the each interconnection prove proper behavior of pseudo-differential filters in analogue signal processing.
\end{abstract}

Keywords-Pseudo-differential filter, current mode, current conveyor

\section{INTRODUCTION}

The present time puts significant emphasis on the development of digital technology, especially in the signal processing area. In this sense, the analogue signal processing may be seen as to be neglected. However, it is necessary to realize that there are permanently analogue signals around us. Therefore, it is inevitable that in signal processing the analogue function blocks are and in the future still will be used. The frequency filters are probably the most discussed function blocks being designed for analogue signal processing. Due to the high demands towards low-voltage and low-power applications next to new or improved types of active elements the interest in the design of fully differential filters [1] - [3] is growing once compared to the basic single-ended counterparts [4]. The reasons may be seen in the advantage of rejecting the common-mode noise signals, feature higher capability to reject power supply noise or enhance dynamic range along with reduced harmonic distortion of the signal being processed. On the other hand, the fully differential filters exhibit generally double circuit complexity compared to single-ended solutions.

To reduce the circuit complexity but still maintain the main features of fully-differential filters, the pseudodifferential filters have emerged, of which to some we are pointed out in the previous two years e.g. [5] - [9]. These filters assume differential input and output but the inner structure is rather single-ended.

For the design of frequency filters (single-ended, fullydifferential or pseudo-differential) different types of active elements are used. As the example, the operational

The research described in this paper was financed by the National Sustainability Program under grant LO1401 and by the Czech Science Foundation under grant no. 16-11460Y. For the research, the infrastructure of the SIX Center was used. transconductance amplifiers (OTAs) [10], current differential transconductance amplifiers (CDTAs) [11] or elements from the family of the current conveyors (CCs) [12] - [18] can be mentioned.

In this paper, we deal with the design of pseudodifferential filters working in the current-mode, whereas the proper operability is verified by analyzing the performance of a fourth-order pseudo-differential frequency filter implemented by means of a cascade connection of two second-order basic blocks. The second-order blocks use three $\mathrm{CCs}$, namely differential voltage current conveyor (DVCC), differential difference current conveyor (DDCC) and second generation current conveyor (CCII) and four passive elements: two resistors ant two capacitors. In theory, the output impedance of the second-order filter (and hence also of the fourth-order filter) is infinitely high and hence it is suitable for easy cascading. The cascade interconnection has been verified in four individual solutions. The pseudodifferential filter is able to realize two basic filtering functions, i.e. low-pass and high-pass response. The provided and discussed simulation results prove the functionality of each cascade interconnection and support the theory of pseudo-differential filters.

\section{DESCRIPTION OF DVCC, DDCC AND CCII}

For the design of pseudo-differential filter operating in current mode the DVCC, DDCC and CCII active elements have been used, which schematic symbols are shown in Fig. 1. and Fig. 2.

DVCC is a five terminal building block with two highimpedance voltage inputs $\mathrm{Y}_{1}$ and $\mathrm{Y}_{2}$, one low-impedance current input $X$, and two high-impedance current outputs $Z_{1+}$ and $Z_{1}$. Relation between the terminal currents and voltages is described by [19]:

$$
V_{\mathrm{x}}=V_{\mathrm{y} 1}-V_{\mathrm{y} 2}, I_{\mathrm{y} 1}=I_{\mathrm{y} 2}=0, I_{\mathrm{z} 1+}=I_{\mathrm{x}}, I_{\mathrm{z} 1-}=-I_{\mathrm{x}}
$$$$
(1 \mathrm{a}, \mathrm{b}, \mathrm{c})
$$

The DDCC is a six-terminal building block with three high-impedance voltage inputs $\mathrm{Y}_{1+}, \mathrm{Y}_{2-}$ and $\mathrm{Y}_{3+}$, a lowimpedance current input $X$, and two high-impedance current outputs $\mathrm{Z}_{1+}$ and $\mathrm{Z}_{1 \text {-. }}$ The relation between terminal currents and voltages is given as [19]:

$$
\begin{gathered}
V_{\mathrm{x}}=V_{\mathrm{y} 1+}-V_{\mathrm{y} 2-}+V_{\mathrm{y} 3+}, I_{\mathrm{y} 1+}=I_{\mathrm{y} 2-}=I_{\mathrm{y} 3+}=0, \\
I_{\mathrm{z} 1+}=I_{\mathrm{x}}, I_{\mathrm{z} 1-}=-I_{\mathrm{x}} . \quad(2 \mathrm{a}, \mathrm{b}, \mathrm{c})
\end{gathered}
$$




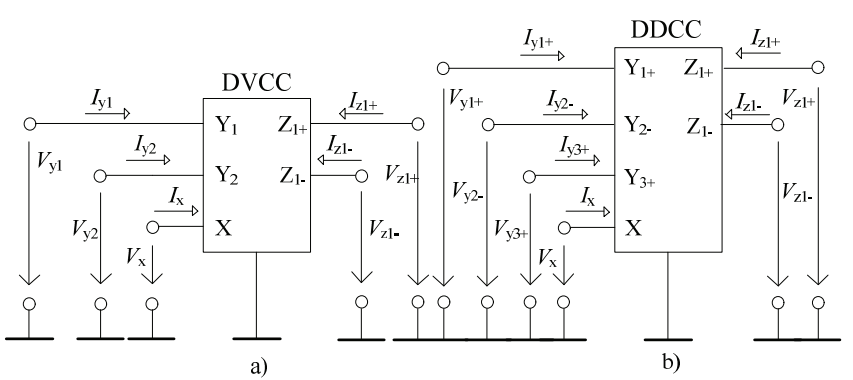

Fig. 1. Schematic symbols of a) DVCC, b) DDCC

The CCII is a three-terminal building block with one high-impedance voltage input $\mathrm{Y}$ and low-impedance current input $X$, and one high-impedance current output $Z$.

The relation between terminal currents and voltages is given as [19]:

$$
V_{\mathrm{x}}=V_{\mathrm{y}}, I_{\mathrm{y}}=0, I_{\mathrm{z}}=I_{\mathrm{x}} .
$$

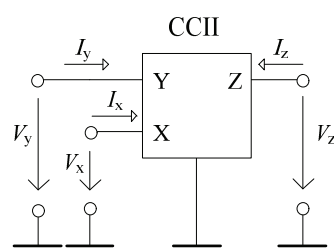

Fig. 2. Schematic symbol of CCII

\section{PSEUDO-DIFFERENTIAL FILTERS}

\section{A. Basic mathematics}

Generally, when analyzing the pseudo-differential circuits operating in current mode, the following relations are assumed:

$$
i_{1 \mathrm{~d}}=i_{1+}-i_{1-}, i_{2 \mathrm{~d}}=i_{2+}-i_{2-},
$$

where $i_{1 \mathrm{~d}}$ and $i_{2 \mathrm{~d}}$ denotes the differential input current and the differential output current, respectively. Signal $i_{1 \mathrm{~d}}$ is the difference between the two input signals $i_{1+}$ and $i_{1 .}$. Signal $i_{2 \mathrm{~d}}$ is the difference between the two output signals $i_{2+}$ and $i_{2-}$ . Taking into consideration (4), then current transfer $K_{\mathrm{i}}$ is defined as follows:

$$
K_{\mathrm{i}}=\frac{i_{2+}-i_{2-}}{i_{1+}-i_{1-}} .
$$

From the mathematical description point of view using (4), (5) it may become obvious that for sake of analysis of such function blocks only the input and output signals are considered. As mentioned in the section I., it is therefore possible to describe and propose so-called pseudodifferential structures, which have differential input and output current terminals, however, the inner circuit topology is single-ended. Thus in practice pseudo-differential filter with single-ended and hence less complicated internal structure may still exhibit high performance comparable to fully-differential structures.

\section{B. Transformation of single-ended input to pseudo- differential in current mode}

The transformation of single-ended input/output voltagemode terminals was already discussed in [6]. Here, we deal the transformation of the current input and output terminals of the filter.

\section{1) Transformation using passive elements}

The first way is to modify the single-ended input to pseudo-differential by means of determined node from the overall solution in Fig. 3.

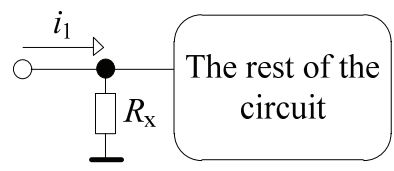

Fig. 3. Single-ended input in current mode with determined node

When converting a single-ended structure into a pseudodifferential, a combination of input nodes are to be determined that results in the required frequency response at the output of the filter, whereas the two input currents are assumed to be shifted by 180 degrees. The situation is described in Fig. 4., where the current $i_{1+}$ is fed to the node with the resistor $R_{\mathrm{x}}$ and the current $i_{1}$ is fed to the node with the resistor $R_{\mathrm{y}}$.

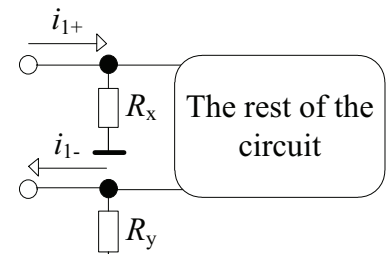

Fig. 4. Pseudo-differential input in current mode with determined node, where $i_{1+}=-i_{1}$

2) Transformation using current conveyors

For transformation by means of current conveyors, such starting structures are suitable, where the input signal is fed directly to the current gate $\mathrm{X}$ of the active element, where high-impedance gate $\mathrm{Y}$ terminals are grounded, for the demonstration single-ended input CCII was used, see Fig. 5 and for the demonstration pseudo-differential input (CCII 1 and CCII 2) was used, see Fig. 6.

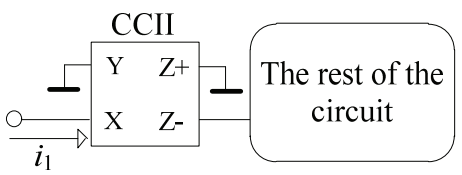

Fig. 5. Single-ended input in current mode with determined CCII

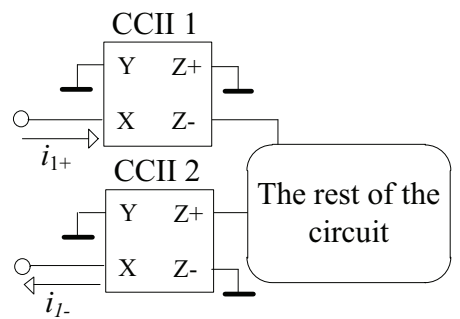

Fig. 6. Pseudo-differential input in current mode with determined CCII 
It is also possible to obtain a pseudo-differential input in the current mode by combining the above described options.

\section{Transformation of single-ended output to pseudo- differential in current mode}

\section{1) Transformation using two current conveyors}

The method of modifications the asymmetric output to symmetric will be demonstration by the current conveyor second generation CCII, these active elements will be determined from the total circuit design.

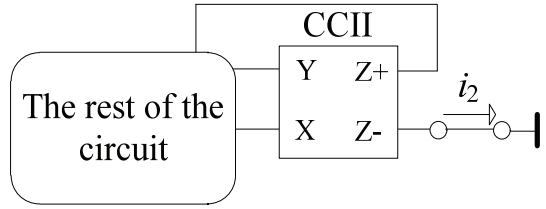

Fig. 7. Single-ended output in current mode with determined CCII

The single-ended output in the current mode demonstrates Fig. 7., where the active CCII element is determined from the total circuit design, the rest of the circuit remains unchanged. Single-ended current output is carried from gate Z-. One way to get a differential output is to extract the differential output current from two active elements. Again the condition is that individual output currents must be phase shifted by 180 degrees, for illustration two current conveyors (CCII 1 and CCII 2) are used as shown in Fig. 8.

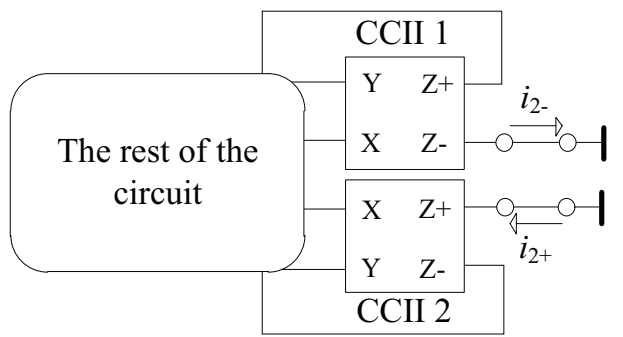

Fig. 8. Pseudo-differential output in current mode with determined two CCIIs

\section{2) Transformation using one current conveyor}

Another and generally more simple possibility to obtain a differential output can be reached by replacing the CCII with an element which, after the same connection as in the case of a single-ended structure, will have two highimpedance outputs of opposite polarity, i.e. Z+ and Z-. The condition for obtaining the pseudo-differential output consists of the two output currents which are in the phase shift of 180 degrees. This situation is illustrated in Fig. 9., where DDCC is determined from the total circuit design. The default single-ended structure remained the same as assumed in Fig. 7.

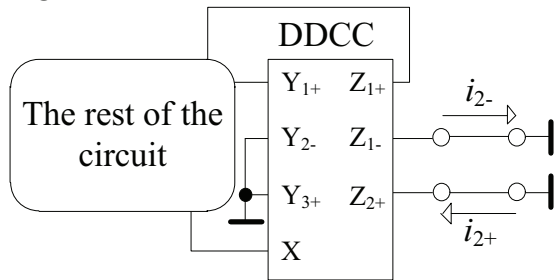

Fig. 9. Pseudo-differential output in current mode with determined DVCC

\section{PROPOSED PSEUDO-DIFFERENTIAL FILTERS}

1) Pseudo-differential filter: basic second-order block Pseudo-differential filter is realized using three active elements, i.e. one DVCC1, one DDCC2 and one CCII-3 and four passive elements: two grounded capacitors and two resistors. This filter uses known $\mathrm{Z}-\mathrm{X}$ connection of the conveyors. Terminals $\mathrm{Y}_{\mathrm{x}}$ are grounded for all current conveyors. Frequency responses are directly taken from the current outputs of the active elements, which ensures high output impedance and thus positive behavior in case of cascade connection.

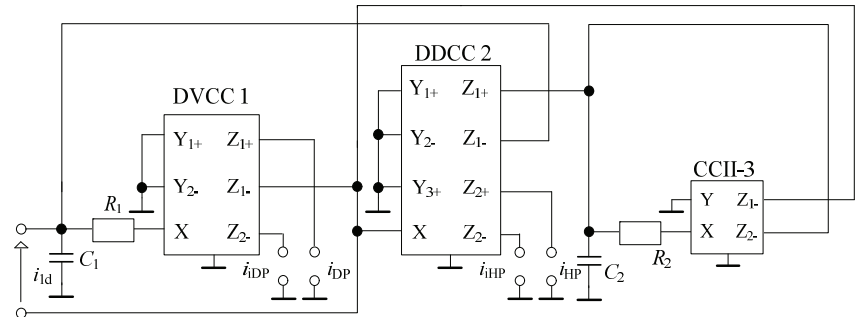

Fig. 10. Pseudo-differential second-order filter operating in current mode

Circuit given in Fig. 10. allows to realize two basic pseudo-differential second-order frequency filter responses, i.e. low- and high-pass. For instance, the positive and negative output currents (6a) and (6b) result in differential high-pass filter response (6c), similarly the low-pass is expressed by (7):

$$
\begin{aligned}
& i_{\mathrm{HP}}=\frac{s^{2} C_{1} C_{2} R_{1} R_{2}}{s^{2} C_{1} C_{2} R_{1} R_{2}+s C_{1} R_{1}+1}, \\
& i_{\mathrm{iHP}}=\frac{-s^{2} C_{1} C_{2} R_{1} R_{2}}{s^{2} C_{1} C_{2} R_{1} R_{2}+s C_{1} R_{1}+1}, \\
& i_{\mathrm{HPd}}=\frac{2 s^{2} C_{1} C_{2} R_{1} R_{2}}{s^{2} C_{1} C_{2} R_{1} R_{2}+s C_{1} R_{1}+1}, \\
& i_{\mathrm{DPd}}=\frac{2}{s^{2} C_{1} C_{2} R_{1} R_{2}+s C_{1} R_{1}+1} .
\end{aligned}
$$

The proposed second-order pseudo-differential frequency filter has the characteristic pole-frequency $\omega_{0}$ and quality factor $Q$ defined as:

$$
\omega_{0}=\frac{1}{\sqrt{C_{1} C_{2} R_{1} R_{2}}}, Q=\sqrt{\frac{C_{2} R_{2}}{C_{1} R_{1}}} .
$$

\section{2) Pseudo-differential filter: fourth-order solution}

The proposed pseudo-differential fourth-order frequency filters operating in current mode were implemented as cascade connection of two basic second-order blocks from Fig. 10. To prove the operability of the final filter, the partial second-order pseudo-differential filters were interconnected in four different ways. Individual types of general cascade interconnection are demonstrated in Fig. 11. - Fig. 14. Two types represent a general cascade connection, 
and two types use decoupling active elements: current controlled current sources (CCCSs).

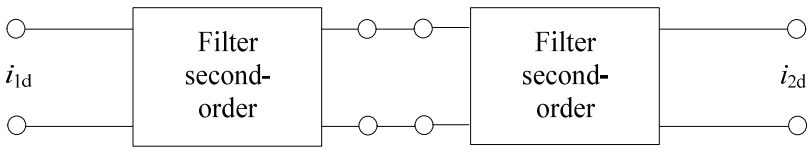

Fig. 11. General cascade connection of the pseudo-differential filter (direct)

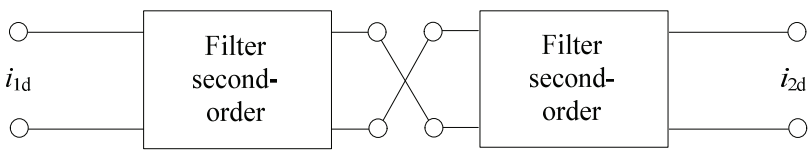

Fig. 12. General cascade connection of the pseudo-differential filter (cross)

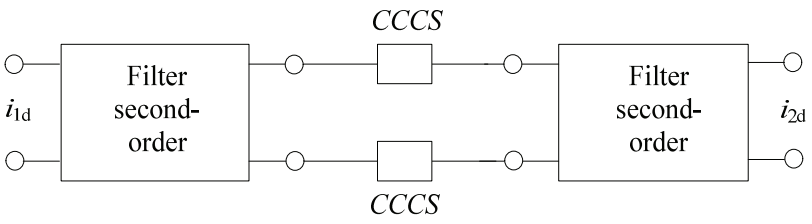

Fig. 13. Cascade connection of the pseudo-differential filter using CCCS (direct)

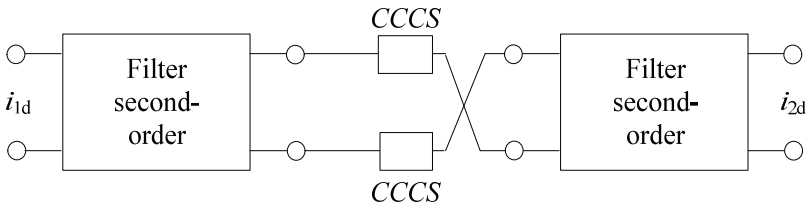

Fig. 14. Cascade connection of the pseudo-differential filter using CCCS (cross)

For both filtration functions (low-pass and high-pass), which the pseudo-differential filters perform, all types of cascade structure from Fig. 11. - Fig. 14. were used and were subject of simulation analysis. The low- and high-pass responses of the fourth-order pseudo-differential filter operating in the current mode can be described as:

$$
\begin{aligned}
i_{\text {DPd }}= & \frac{2}{s^{2} C_{21} C_{22} R_{21} R_{22}+s C_{11} R_{11}+1} . \\
& \frac{2}{s^{2} C_{21} C_{22} R_{21} R_{22}+s C_{21} R_{21}+1} \\
i_{\mathrm{HPd}}= & \frac{2 s^{2} C_{11} C_{12} R_{11} R_{12}}{s^{2} C_{11} C_{12} R_{11} R_{12}+s C_{11} R_{11}+1} \\
& \cdot \frac{2 s^{2} C_{11} C_{12} R_{11} R_{12}}{s^{2} C_{11} C_{12} R_{11} R_{12}+s C_{21} R_{21}+1}
\end{aligned}
$$

\section{SimUlations RESUlTS OF THE FILTERS}

Butterworth coefficients were used for the calculation. These coefficients have been matched to the mathematical description.

1) Simulations of second-order pseudo-differential filter For simulations the values of capacitors $C_{1}=C_{2}=1 \mathrm{nF}$ were selected and the values of resistors were determined as $R_{1}=2251 \Omega$ and $R_{2}=1125 \Omega$ to obtain the pole-frequency $100 \mathrm{kHz}$ and quality factor $Q=0.707$. The active elements in the circuit topology were modeled using the model of the UCC-N1B integrated circuit [20].

The performance of the filter has been verified by simulations, where the obtained frequency responses of the pseudo-differential second-order filter working in current mode are shown in Fig. 15. and Fig. 16.

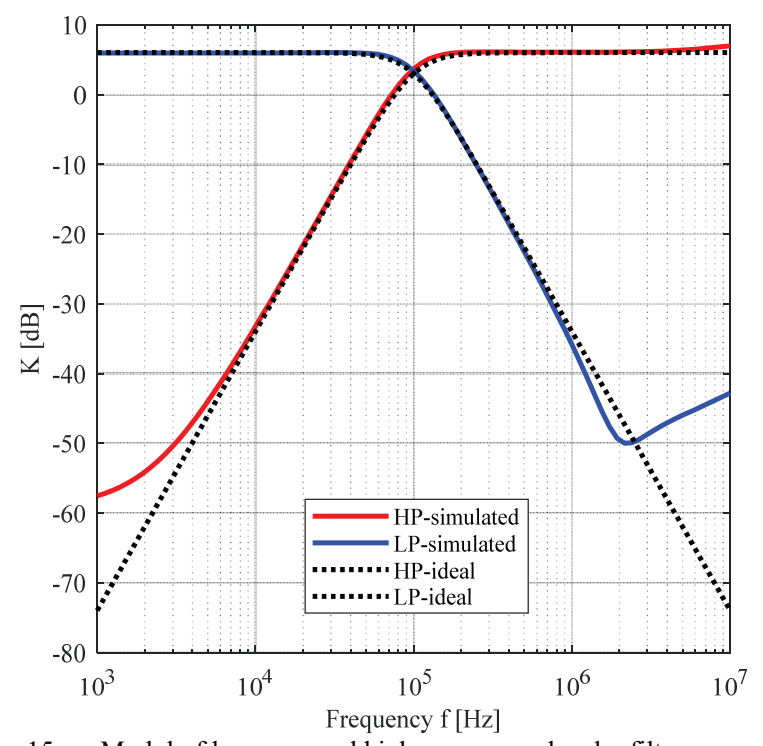

Fig. 15. Modul of low-pass and high-pass second-order filters

It is clear from the graph that the filter of the high-pass and low-pass filter is very close to the ideal state. Better results of the modul characteristic are achieved by the highpass through, where the reached attenuation is approximately $58 \mathrm{~dB}$. Low-pass filter reached the maximum attenuation of $43 \mathrm{~dB}$ and the increase of the magnitude of the low-pass filter at the frequency of approximately 1.1 $\mathrm{MHz}$ is caused by the non-ideal behavior of the active elements used in simulations.

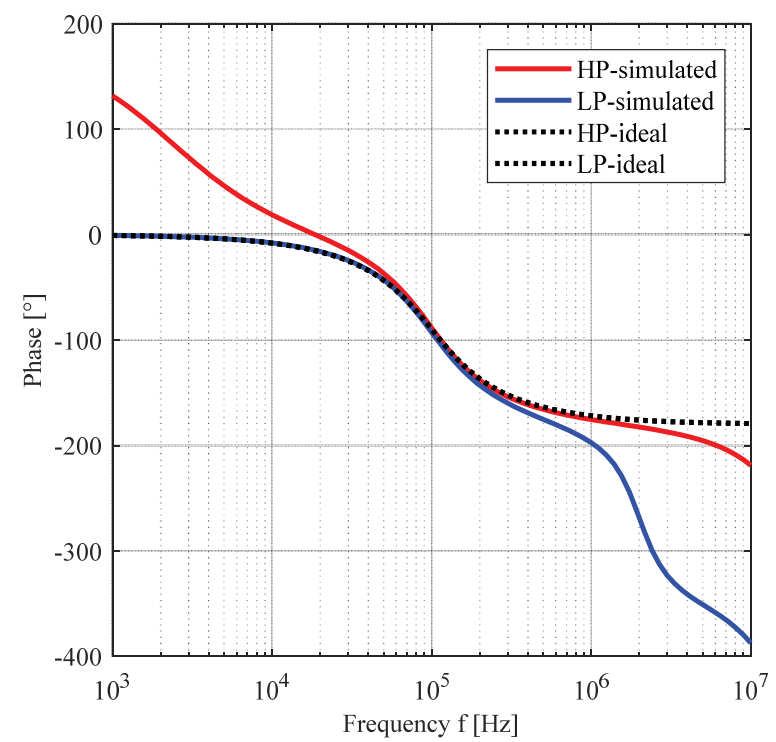

Fig. 16. Phase characteristics low-pass and high-pass second-order filter 
The phase shift of the high-pass transfer starts at approximately 120 degrees, the ideal state was attained only around pole-frequency. At the low-pass filter on lower frequencies was achieved nearly ideal characteristics, waveform deteriorates after pole-frequency.

\section{2) Simulations of fourth-order pseudo-differential filter}

The fourth-order pseudo-differential filter operating in the current mode was designed as a cascade connection of the basic second-order solution from Fig. 10. For simulation purposes, Butterworth approximation was considered again. Also here, the values of capacitors $C_{11}=C_{12}=C_{21}=C_{22}=$ $1 \mathrm{nF}$ were selected and the resistor values $R_{11}=1218 \Omega, R_{12}$ $=2080 \Omega, R_{21}=2942 \Omega, R_{22}=862 \Omega$ were calculated resulting in $100 \mathrm{kHz}$ pole-frequency. The plots in Fig. 17. and Fig. 18. show modul and phase characteristics for all four different ways of interconnection from Fig. 11. to Fig. 14.

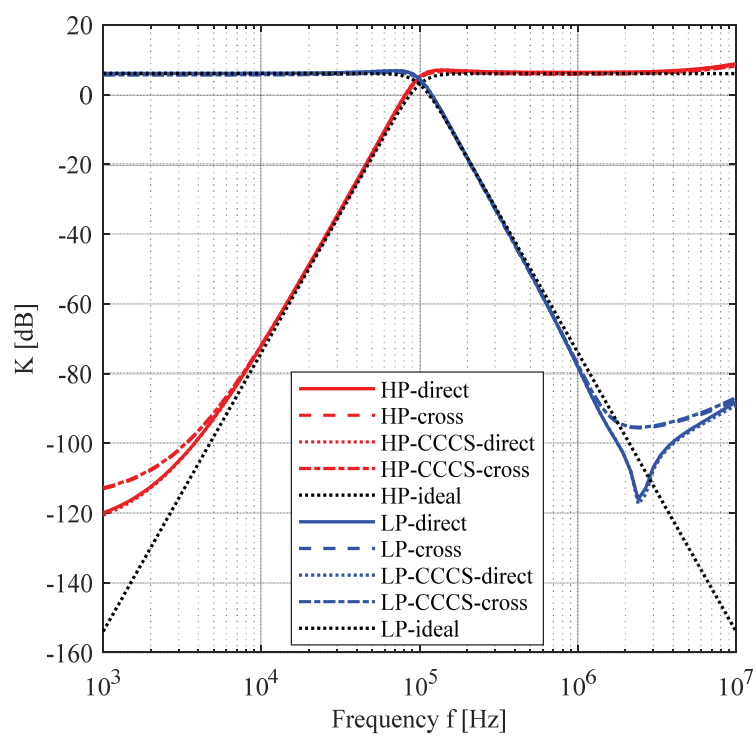

Fig. 17. Modules of low-pass and high-pass fourth-order filters

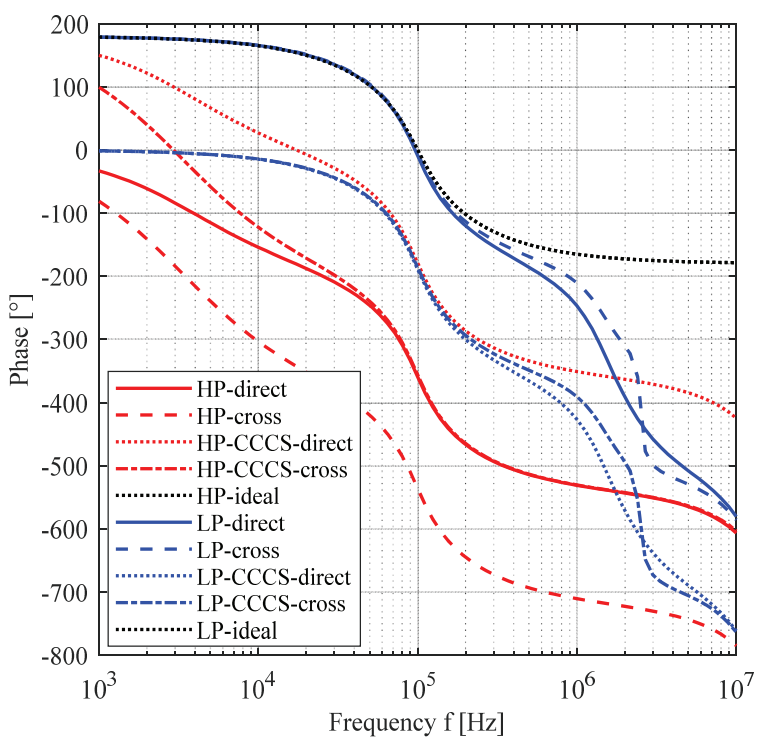

Fig. 18. Phase responses of low-pass and high-pass fourth-order filters

From the modul characteristics, it is clear that the fourthorder high-pass filter has almost ideal properties, better results are achieved by the general direct-connection from Fig. 11. and direct-connection using CCCS according to Fig. 13. with attenuation of approximately $120 \mathrm{~dB}$. The cross-connection from Fig. 12. and cross-connection using CCCS from Fig. 14 also has a very high-quality result, just compared to the direct-connections the attenuation is about $5 \mathrm{~dB}$ smaller. For the fourth-order filter, the error was even more reflected on the $1.1 \mathrm{MHz}$ frequency. The better results were achieved by direct-connections in Fig. 12. and Fig. 14.

The phase characteristics, the greatest differences in the results for both transfer functions are apparent. The lowpass filter for both connections without the use of the CCCS (LP-direct, LP-cross), but the transfer function deteriorates and falls sharply after pole-frequency. High-pass filter shows the best results at the HP-CCCS-direct, but it has to be said that to the ideal transfer is only approaching. Smaller the imperfections are caused by real properties of the active elements emulated by the UCC_N1B [20] simulation active elements. The best results are both cross-connections, applies to HP and LP.

However, it is necessary to say that the pseudodifferential filter is fully functional for all four different cascade connections. The fourth-order pseudo-differential filter can be engaged in with no use - CCCS, but only if the conditions are met from the perspective of the highimpedance output or the low-impedance input of the filter. The aim of the article was to verify the functionality of the fourth-order pseudo-differential filter, which was implemented by the cascading interconnection of the two second-order basic blocks.

\section{CONCLUSION}

In this paper the theory of pseudo-differential filters was investigated, where the possibility of designing higher-order filters was analyzed. Using the basic second-order pseudodifferential filter featuring high-impedance outputs connected in cascade, the fourth-order pseudo-differential filter working in the current-mode was designed. The operability of the final filter was supported by analyzing four different interconnections of the two basic blocks. Based on the presented simulation results, it was shown that the theory of pseudo-differential filter is valid. This is proved by achieving generally the same results in frequency response of the filter for the cases if the CCCS decoupling blocks are used or not and for both direct and cross interconnections of the partial second-order blocks. Therefore, it can be stated that the theory of pseudodifferential filters is fully functional also for higher-order solutions and designes.

\section{REFERENCES}

[1] Dvorak, J. Jerabek, J. Langhammer, L. Kapoulea, S. Psychalinos, C. (2018). Design of Fully-Differential Frequency Filter with FractionalOrder Elements. DOI: 10.1109/TSP.2018.8441259.

[2] P. Beg, I. A. Khan, S. Maheshwari, and M. A. Siddiqui, Digi-tally Programmable Fully Differential Filter, Radioengineering 20 (2011) 917-925.)

[3] N. Herencsar, J. Jerabek, J. Koton, K. Vrba, S. Minaei, I. C. Goknar, Pole Frequency and Pass-Band Gain Tunable Novel Fully-Differential Current-Mode All-Pass Filter, IEEE Int. Symposium on Circuits and Systems (ISCAS), May 2015. 
[4] W.-K. Chen, The circuits and fiters handbook, (3rd edition, CRC Press, 2003), p. 2961

[5] O. Sladok, J. Koton, N. Herencsar. Universal Pseudo-Differential Filter Using DDCC and DVCCs. Elektronika Ir Elektrotechnika, 2017, vol. 23, no. 6, p. 46-52. ISSN: 1392-1215. [Online]. Available: http://dx.doi.org/10.5755/j01.eie.23.6.19694

[6] J. Koton, N. Herencsar, O. Sladok, J. Horng, "Pseudo-differential second order band reject filter using current conveyors", AEU International Journal of Electronics and Communications, vol. 70, no. 6, pp. 814-821, 2016. [Online]. Available: http://dx.doi.org/10.1016/j.aeue.2016.03.009

[7] J. Koton, N. Herencsar, J.-W. Horng, "Differential Second-Order Voltage-Mode All-Pass Filter Using Current Conveyors", Elektronika ir Elektrotechnika, vol. 22, no. 5, pp. 52-57, 2016. [Online]. Available: http://dx.doi.org/10.5755/j01.eie.22.5.16344

[8] O. Sladok. Pseudo-Differential Filter Using DDCC. In Proc. 22nd conf. Student EEICT. 2016. s. 147-149. ISBN: 978-80-214-5350- 0.

[9] R. Sotner, N. Herencsar, J. Jerabek, A. Kartci, J. Koton, T. Dostal.. Pseudo-Differential Filter Design Using Novel Adjustable Floating Inductance Simulator with Electronically Controllable Current Conveyors. Elektronika Ir Elektrotechnika, 2017, vol. 23, no. 2, p. 3135. ISSN: 1392-1215.

[10] D. Agrawal, S. Maheshwari, Cascadable current mode instrumentation amplifier, AEU - International Journal of Electronics and Communications, Volume 94, 2018, Pages 91-101, ISSN 14348411, https://doi.org/10.1016/j.aeue.2018.06.038.

[11] M. A. Eldeeb, Y. H. Ghallab, Y. Ismail, H. Elghitani, Low-voltage subthreshold CMOS current mode circuits: Design and applications, AEU - International Journal of Electronics and Communications, Volume 82, 2017, Pages 251-264, ISSN 1434-8411, https://doi.org/10.1016/j.aeue.2017.08.049.

[12] Ch. Hua-Pin, H. Yuh-Shyan, K. Yi-Tsen, A systematic realization of third-order quadrature oscillator with controllable amplitude, AEU International Journal of Electronics and Communications, Volume 79, 2017, Pages 64-73, ISSN 1434-8411,

https://doi.org/10.1016/j.aeue.2017.05.039.
[13] E. Yuce. A single-input multiple-output voltage-mode second-order universal filter using only grounded passive components. Indian Journal of Engineering \& Materials Sciences. Vol. 24, April 2017, pp. 97-106.

[14] P. Farago, L. Festila, S. Hintea, G. Csipkes, D. Csipkes, P. Soser, "A Transistor-Level Reconfigurable Circuit for Rapid Transconductor Design and Testing", Elektronika ir Elektrotechnika, vol. 117, no. 1, pp. 99-104, 2012 [Online].

[15] F. Kacar, S. Menekay, "Realization of a CMOS Current Differencing Buffer Amplifier and its Filter Application", in Proc. The 7th International Conference Electrical and Electronics Engineering (ELECO), Bursa, Turkey, 2011, pp. 337-340.

[16] H. Chen, "Current-mode dual-output ICCII-based tunable universal biquadratic filter with low-input and high output impedances", International Journal of Circuit Theory and Applications, vol. 42, Issue 4, pp. 376-393, 2012. [Online]. Available: http://dx.doi.org/10.1002/cta.1858

[17] J.-W.Horng, W.-Y. Chiu, T.-Y. Teng, C.-H. Lin, N. Herencsar, J. Koton, Cascadable voltage- mode three inputs universal biquad using DVCCs. In Proc. of 2014 14th International Symposium on Communications and Information Technologies (ISCIT). 2014.s. 299-303. ISBN: 978-1-4799-4416- 3.

[18] B. Metin, N. Herencsar, J. Koton, E. Arslan. All- pass filter application using electronically tunable DDCC. In Proceedings of the 24th International Conference Radioelektronika 2014. Bratislava: 2014. s. 1-4. ISBN: 978-1-4799-3714- 1

[19] R. Senani, D. R. Bhaskar, A. K. Singh, Current Conveyors: Variant,Applications and Hardware Implementations. Switzerland: Springer-Verlag, 2015. [Online]. Available: http://dx.doi.org/10.1007/978-3-319-08684-2

[20] Datasheet UCC-N1B 0520. Universal current conveyor (UCC) and second-generation current conveyor $(\mathrm{CCII}+/-)$, rev. 1. Brno University of Technology, On Semiconductor Ltd.; 2012. 